\title{
Allelopathic effect of Callistemon lanceolatus DC. against two cultivars of Triticum aestivum $\mathbf{L}$.
}

\author{
Nazia Shah, Imtiaz Ahmad, Muhammad Imran*, Hina Gul, \\ Muhammad Jamal Babar, Waqar Ali Shah and Sakina Anwar \\ Department of Botany, Bacha Khan University, Charsadda-Pakistan \\ *Corresponding author's email: imranbotany12345@gmail.com \\ Citation
}

Nazia Shah, Imtiaz Ahmad, Muhammad Imran, Hina Gul, Muhammad Jamal Babar, Waqar Ali Shah and

Sakina Anwar. Allelopathic effect of Callistemon lanceolatus DC. against two cultivars of Triticum aestivum L. Pure and Applied Biology. Vol. 7, Issue 2, pp783-790. http://dx.doi.org/10.19045/bspab.2018.70097

\begin{tabular}{llll}
\hline \hline Received: 17/03/2018 & Revised: 05/06/2018 & Accepted: 21/06/2018 & Online First: 28/06/2018 \\
\hline \hline
\end{tabular}

\section{Abstract}

Generally, the plants have effects on the other plants growing in their vicinity. The plants may have both positive and negative effects on each other. The purposes of the current study were to assess the allelopathic capability of Callistemon lanceolatus against the two cultivars of Triticum aestivum, under laboratory condition. Allelopathic potential of the plant was determined using aqueous extracts of various parts of the plant. Aqueous extract of leaves and bark were acquired by independently soaking $5 \mathrm{gm}$ and $10 \mathrm{gm}$ powder in $100 \mathrm{ml}$ distilled water for 24, 48 and 72 hours. The results revealed that allelopathic potential of the plant was both dose and soaking duration dependent. Generally, extract of higher concentration i.e. $10 \mathrm{gm} / 100 \mathrm{ml}$ soaked for higher duration ( 72 hours) were more inhibitory than lower concentrations (5gm/100 and shorter soaking duration 24 and 48 hours). Hot water extracts, Litter and mulches of the plant also significantly repressed seed germination and the general development of both the test crops. Seminal roots numbers were also suppressed by all the treatments used in the study. It was concluded that the plant has strong allelopathic potential. Further investigation is required to isolate the chemicals that triggered inhibition which may provide base to for the development of novel synthetic pesticides. The study further suggests that cultivation of the plant should be avoided in the vicinity of the crop fields.

Keywords: Allelopathy; Aqueous extract; Callistemon lanceolatus; Phytotoxicity; Triticum aestivum

\section{Introduction}

Allelopathy is a mechanism in which chemicals produced by the plants may increase or decrease the growth of associated plants. Plants live in associations relying on the biological necessities. In nature whenever two or more than two species live in the same place, they are dependent on each other for numerous requirements for their growth [1]. Allelopathy is a complex characteristic process that activates along with rivalry to supress lastly eliminate susceptible related species from the common environment [ 2 , 3]. Allelopathy can likewise be a valuable natural as well as biological control agent. $[4,5]$. Recent research work identified a number of allelopathic plant species including Imperata Clindrica [6], Anagllis arvensis [7], Accacia nilotica and Eucalyptus rostrate [8], Parthenium hysterophorus [9], Ficus infectoria, Prospis juliflora, Emblica officinalis and Acacia leucophloea [10] and Jatropha 
curcas [11]. Similarly, the allelopathy of Vitex negundo [12], Sorghum vulgar, Nerium oleander, Datura inoxia, Nicotiana tabacum, Ricinus communis and Crocus sativus [13], Celtis australis [14] and Leucaena leucocephala [15] has also been worked out. [16] investigated Cassia angustifolia for its allelopathic potential. [17] tested the phytotoxcity of Brachiaria decumbens. [18] reported that Cenchrus ciliarus and Bothriochloa pertusa exhibit allelopathy.

Callistemon lanceolatus DC. commonly called Bottle brushes tree belong to the family Myrtaceae. C. lanceolatus is a small tree native to east and south-east of Australia and New Zealand, tropical Asia (Pakistan, Sarilanka and Nepal) temperate Asia (Japan, China, Russia), they frequently grow in soggy or wet environments, for example, along stream beds or in territories which are inclined to surges. They also cultivated as ornamentally in the gardens and also along with the fields in Charsadda. Therefore, the current investigations were made to evaluate the potential of $C$. lanceolatus against two different varieties of wheat.

\section{Materials and methods}

The freshly bark and leaves of the $C$. lanceolatus were collected and kept in shade at room temperature $\left(25-30{ }^{0} \mathrm{C}\right)$ for drying. The dried material was then grinded into fine powder. The grinded powder of the plant parts was kept in small plastic bags for further research activities. Certified seed of two wheat cultivars viz., Millat and Faiz Abad were obtained from authorized dealer.

\section{Aqueous extract bioassay}

The powder of the plant in the amount of $5 \mathrm{gm}$ and $10 \mathrm{gm}$ were separately soaked in $100 \mathrm{ml}$ distilled water, for shorter (24), medium (48) and longer (72) hours' durations. For the aqueous extract bioassays, the Petri dishes were used. The two folds' filter paper moistened with the respective extract were kept in the test petri plates while the same fold filter paper moistened with distilled water used as control. Each petri plate contained uniformly placed 10 seeds of test crops. The percent germination, radicle, plumule growth, fresh, dry weight, moisture contents and number of seminal roots were recorded after 72 hours. Similarly, in hot aqueous extract bioassay the same procedure was followed but extract acquired through temperature.

\section{Mulching bioassay}

The sterilized sand was mixed with $5 \mathrm{gm}$ and $10 \mathrm{gm}$ of powder. The small plastic pots were filled with the mixture of powder and sand. Ten seeds of both the verities of test crop species were sown in each pot separately, 5 replicates were made for each treatment. Fine pieces of filter paper were used instead of powder to retain moisture contents in the control treatments. The same data were recorded as for aqueous extract bioassay after 7 days of sowing the seeds.

Litter bioassay: The grinded powder of both the parts of plant were mixed and placed in the petri plates, topped with a single filter paper which was moistened with water. Double sheet of filter paper was used without powder material in the control treatments. Ten seeds of both test species were placed above the filter paper at uniform distance. Five replicates were made for each treatment. The data were recorded after 7 days [14]. All the collected data were recorded and analysed through one-way ANOVA using SPSS 17 version.

\section{Results and discussion \\ Effect of cold aqueous extracts}

The recorded and analyzed data of the aqueous extracts showed significant inhibition in germination, radicle and plumule growth, fresh and dry weight, moisture contents and seminal roots number of both the test crop species. It was observed that higher concentration $(10 \mathrm{gm} / 100 \mathrm{ml})$ with longer soaking duration 72 hours showed maximum inhibition as compared to low concentrations $(5 \mathrm{gm} / 100 \mathrm{ml})$ and shorter (24 hours) and medium (48 hours) soaking 
duration respectively. The most susceptible test variety of wheat to all the extracts was found to be F. Abad. The hot water extract was also proven to be inhibitory to the test species. It was also recorded that the cold extract showed more reduction as compared to hot water extract. Maximum inhibition was recorded for leaves extract as compared to bark (Table 1-4).

Table 1. Effect of aqueous extract of $C$. Lanceolatus on germination of wheat verities

\begin{tabular}{|c|c|c|}
\hline \multirow{2}{*}{ Treatments/ extracts } & \multicolumn{2}{|c|}{ Test species } \\
\cline { 2 - 3 } & $\begin{array}{c}\text { T. aestivum } \\
\text { (Cultivar Millat) }\end{array}$ & $\begin{array}{c}\text { T. aestivum } \\
\text { (Cultivar F. Abad) }\end{array}$ \\
\cline { 2 - 3 } Control & \% Germination & \% Germination \\
\hline $5 \mathrm{gm} / 100 \mathrm{ml}$ leaf $24 \mathrm{hrs}$. & $100.00 \pm 0.00$ & $100.00 \pm 0.00$ \\
\hline $5 \mathrm{gm} / 100 \mathrm{ml}$ leaf $48 \mathrm{hrs}$. & $73.33 \pm 0.43^{*}$ & $66.70 \pm 0.53^{*}$ \\
\hline $5 \mathrm{gm} / 100 \mathrm{ml}$ leaf $72 \mathrm{hrs}$. & $70.10 \pm 0.18^{*}$ & $60.00 \pm 0.08^{*}$ \\
\hline $10 \mathrm{gm} / 100 \mathrm{ml}$ leaf $24 \mathrm{hrs}$. & $69.00 \pm 0.43^{*}$ & $53.70 \pm 0.01^{*}$ \\
\hline $10 \mathrm{gm} / 100 \mathrm{ml}$ leaf $48 \mathrm{hrs}$. & $66.06 \pm 0.11^{*}$ & $56.70 \pm 0.03^{*}$ \\
\hline $10 \mathrm{gm} / 100 \mathrm{ml}$ leaf $72 \mathrm{hrs}$. & $60.30 \pm 0.01^{*}$ & $50.00 \pm 0.00^{*}$ \\
\hline $5 \mathrm{gm} / 100 \mathrm{ml}$ bark $24 \mathrm{hrs}$. & $50.70 \pm 0.00^{*}$ & $45.00 \pm 0.00^{*}$ \\
\hline $5 \mathrm{gm} / 100 \mathrm{ml}$ bark 48 hrs. & $83.05 \pm 1.00^{*}$ & $83.30 \pm 1.00^{*}$ \\
\hline $5 \mathrm{gm} / 100 \mathrm{ml}$ bark $72 \mathrm{hrs}$. & $81.10 \pm 1.00^{*}$ & $80.30 \pm 0.94^{*}$ \\
\hline $10 \mathrm{gm} / 100 \mathrm{ml}$ bark $24 \mathrm{hrs}$. & $78.53 \pm 1.00^{*}$ & $79.30 \pm 1.00^{*}$ \\
\hline $10 \mathrm{gm} / 100 \mathrm{ml}$ bark $48 \mathrm{hrs}$. & $77.40 \pm 1.00^{*}$ & $76.70 \pm 0.46^{*}$ \\
\hline $10 \mathrm{gm} / 100 \mathrm{ml}$ bark 72 hrs. & $75.02 \pm 1.00^{*}$ & $66.70 \pm 0.22^{*}$ \\
\hline $5 \mathrm{~g} / 100 \mathrm{ml}$ hot water leaf extract & $75.08 \pm 1.00^{*}$ & $87.00 \pm 0.70^{*}$ \\
\hline $10 \mathrm{~g} / 100 \mathrm{ml}$ hot water leaf extract & $78.00 \pm 0.92^{*}$ & $50.00 \pm 0.02^{*}$ \\
\hline $5 \mathrm{~g} / 100 \mathrm{ml}$ hot water bark extract & $76.00 \pm 0.11^{*}$ & $70.00 \pm 0.92^{*}$ \\
\hline $10 \mathrm{~g} / 100 \mathrm{ml}$ hot water bark extract & $80.00 \pm 1.00^{*}$ & $67.00 \pm 0.99^{*}$ \\
\hline
\end{tabular}

Each value is the grand mean of five replicates each having ten seeds. Ns $=$ Non significant, $*=$ Significant at $\alpha$ $<0.05$

The results are in agreement with those of [19-22] who also reported reduction in wheat seed germination after extracts application. Our results also agree with those of $[2,3,23-25]$, in this aspects who also reported similar allelopathic behaviour of some plants. Our finding also agrees with those of [9] who's reported aqueous extracts of Parthenium hysterophorus, Eucalyptus camaldulensis [26] and Dodonaea viscosa [27] had significantly inhibitory effects on plumule and radical length. In the present study the seminal roots number also significantly reduced by both hot and cold extract in same manner as that higher concentration extract soaked for longer duration triggered maximum reduction (Table 4). Hot water extracts also showed inhibitory effects on both test species. [14] also reported hot water extracts to be inhibitory in contradiction of test species. Fresh, dry weight and moisture contents of both wheat cultivars were significantly affected by both leaves and bark aqueous extracts (Table 3). Our findings were agreed with those of $[18,24$, 28,29 ] who's reported similar reduction in fresh and dry weight due to the allelopathic effects of the plants used in the experiments. Similar results were also obtained by [30] after the treatment of rice plant with allelopathic phenolic compounds. [31] reported reduction in fresh weight of pearl millet and cowpea due to extracts application. Other researcher also reported the same manner of inhibition in case of moisture contents of 
Table 2. Effect of aqueous extracts of $C$. lanceolatus on Radicle and plumule length of wheat verities

\begin{tabular}{|c|c|c|c|c|}
\hline \multirow{2}{*}{ Treatments/ extracts } & \multicolumn{3}{|c|}{$\begin{array}{c}\text { Test species } \\
\text { Cultivar Millat) in mm }\end{array}$} & $\begin{array}{c}\text { T. aestivum } \\
\text { (Cultivar F. Abad) in mm }\end{array}$ \\
\cline { 2 - 5 } & Radical & Plumule & Radical & Plumule \\
\hline Control & $75.03 \pm 0.01$ & $79.30 \pm 0.05$ & $65.33 \pm 0.03$ & $68.83 \pm 0.07$ \\
\hline $5 \mathrm{gm} / 100 \mathrm{ml}$ leaf $24 \mathrm{hrs}$. & $12.50 \pm 0.00^{*}$ & $15.90 \pm 0.00^{*}$ & $09.50 \pm 0.00^{*}$ & $06.23 \pm 0.00^{*}$ \\
\hline $5 \mathrm{gm} / 100 \mathrm{ml}$ leaf $48 \mathrm{hrs}$. & $11.50 \pm 0.00^{*}$ & $13.90 \pm 0.00^{*}$ & $08.80 \pm 0.00^{*}$ & $05.80 \pm 0.00^{*}$ \\
\hline $5 \mathrm{gm} / 100 \mathrm{ml}$ leaf $72 \mathrm{hrs}$. & $11.20 \pm 0.00^{*}$ & $11.77 \pm 0.00^{*}$ & $07.83 \pm 0.00^{*}$ & $03.53 \pm 0.00^{*}$ \\
\hline $10 \mathrm{gm} / 100 \mathrm{ml}$ leaf $24 \mathrm{hrs}$. & $09.50 \pm 0.00^{*}$ & $06.33 \pm 0.00^{*}$ & $07.05 \pm 0.00^{*}$ & $03.33 \pm 0.00^{*}$ \\
\hline $10 \mathrm{gm} / 100 \mathrm{ml}$ leaf $48 \mathrm{hrs}$. & $08.57 \pm 0.00^{*}$ & $05.60 \pm 0.00^{*}$ & $06.17 \pm 0.00^{*}$ & $02.27 \pm 0.00^{*}$ \\
\hline $10 \mathrm{gm} / 100 \mathrm{ml}$ leaf 72 hrs. & $07.33 \pm 0.00^{*}$ & $04.87 \pm 0.00^{*}$ & $04.50 \pm 0.00^{*}$ & $02.10 \pm 0.00^{*}$ \\
\hline $5 \mathrm{gm} / 100 \mathrm{ml}$ bark $24 \mathrm{hrs}$. & $32.87 \pm 0.43^{*}$ & $36.10 \pm 1.00^{*}$ & $36.80 \pm 0.00^{*}$ & $24.10 \pm 0.00^{*}$ \\
\hline $5 \mathrm{gm} / 100 \mathrm{ml}$ bark 48 hrs. & $29.17 \pm 0.07^{*}$ & $35.67 \pm 1.00^{*}$ & $27.83 \pm 0.00^{*}$ & $24.00 \pm 0.00^{*}$ \\
\hline $5 \mathrm{gm} / 100 \mathrm{ml}$ bark $72 \mathrm{hrs}$. & $24.43 \pm 0.01^{*}$ & $32.67 \pm 0.03^{*}$ & $26.17 \pm 0.00^{*}$ & $17.40 \pm 0.00^{*}$ \\
\hline $10 \mathrm{gm} / 100 \mathrm{ml}$ bark $24 \mathrm{hrs}$. & $29.43 \pm 0.02^{*}$ & $33.57 \pm 0.05^{*}$ & $26.83 \pm 0.00^{*}$ & $22.40 \pm 0.00^{*}$ \\
\hline $10 \mathrm{gm} / 100 \mathrm{ml}$ bark $48 \mathrm{hrs}$. & $21.33 \pm 0.01^{*}$ & $26.50 \pm 0.04^{*}$ & $25.80 \pm 0.00^{*}$ & $20.87 \pm 0.00^{*}$ \\
\hline $10 \mathrm{gm} / 100 \mathrm{ml}$ bark $72 \mathrm{hrs}$. & $19.70 \pm 0.01^{*}$ & $23.60 \pm 0.03^{*}$ & $23.23 \pm 0.00^{*}$ & $17.03 \pm 0.00^{*}$ \\
\hline $5 \mathrm{~g} / 100 \mathrm{ml}$ hot water leaf extract & $06.67 \pm 0.00^{*}$ & $09.63 \pm 0.01^{*}$ & $14.30 \pm 0.00^{*}$ & $07.67 \pm 0.00^{*}$ \\
\hline $10 \mathrm{~g} / 100 \mathrm{ml}$ hot water leaf extract & $06.47 \pm 0.00^{*}$ & $07.20 \pm 0.01^{*}$ & $03.23 \pm 0.00^{*}$ & $01.77 \pm 0.00^{*}$ \\
\hline $5 \mathrm{~g} / 100 \mathrm{ml}$ hot water bark extract & $27.67 \pm 0.01^{*}$ & $35.90 \pm 0.04^{*}$ & $36.83 \pm 0.00^{*}$ & $16.80 \pm 0.00^{*}$ \\
\hline $10 \mathrm{~g} / 100 \mathrm{ml}$ hot water bark extract & $21.00 \pm 0.01^{*}$ & $26.47 \pm 0.01^{*}$ & $21.93 \pm 0.00^{*}$ & $14.00 \pm 0.00^{*}$ \\
\hline
\end{tabular}

Each value is the grand mean of five replicates each having ten seeds.

Ns $=$ Non-significant, $*=$ Significant at $\alpha<0.05$

\section{Mulching and litter effect}

Added much and litter also showed significant inhibition in both cultivars. It was stated earlier that plant litter usually increases soil fertility during decay but allelopathic plants release phytotoxic substances before decay that cause soil phytotoxicity in natural ecosystems [18]. Plants release phytochemicals in litter and mulch processes, and their assimilation to the soil might support their harmful effect in the field [33]. This feature when verified by using $C$. lanceolatus litter in trials indicated that there was significant inhibition in the growth of the test species. Leaves and bark's mulch inhibited germination of both cultivars of $T$. aestivum. All other parameters including germination, plumule and radical growth, number of seminal root, fresh, dry weight, and moisture contents of both test species also reduced by added litter and mulch (Table 5). Our findings were agreed in case of mulch and litter with those of [14, 34] who's presented the similar differential behaviour of Celtis australis L. and Polypogon monspeliensis L. 
Table 3. Effect of aqueous extracts of $C$. lanceolatuson fresh, dry weight and moisture contents of wheat verities

\begin{tabular}{|c|c|c|c|c|c|c|}
\hline \multirow{4}{*}{ Treatments/ extracts } & \multicolumn{6}{|c|}{ Test species } \\
\hline & \multicolumn{3}{|c|}{$\begin{array}{c}\text { T. aestivum } \\
\text { (Cultivar Millat) }\end{array}$} & \multicolumn{3}{|c|}{$\begin{array}{c}\text { T. aestivum } \\
\text { (Cultivar F. Abad) }\end{array}$} \\
\hline & \multicolumn{6}{|c|}{ Percent of control values } \\
\hline & $\begin{array}{l}\text { Fresh } \\
\text { weight } \\
\text { (in mg) }\end{array}$ & $\begin{array}{c}\text { Dry } \\
\text { weight } \\
\text { (in } \mathbf{~ g g )}\end{array}$ & $\begin{array}{l}\text { Moisture } \\
\text { contents }\end{array}$ & $\begin{array}{c}\text { Fresh } \\
\text { weight } \\
\text { (in } \mathrm{mg} \text { ) }\end{array}$ & 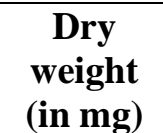 & $\begin{array}{l}\text { Moisture } \\
\text { contents }\end{array}$ \\
\hline $5 \mathrm{gm} / 100 \mathrm{ml}$ leaf $24 \mathrm{hrs}$. & $60.37^{*}$ & $51.95^{*}$ & $41.10 *$ & $62.76^{*}$ & $46.07^{*}$ & $54.95 *$ \\
\hline $5 \mathrm{gm} / 100 \mathrm{ml}$ leaf $48 \mathrm{hrs}$. & $56.72 *$ & $49.35 *$ & $33.97 *$ & $61.38^{*}$ & $46.07 *$ & $51.34 *$ \\
\hline $5 \mathrm{gm} / 100 \mathrm{ml}$ leaf $72 \mathrm{hrs}$. & $55.97 *$ & $41.56^{*}$ & $42.24 *$ & $60.00 *$ & $44.94 *$ & $49.06^{*}$ \\
\hline $10 \mathrm{gm} / 100 \mathrm{ml}$ leaf $24 \mathrm{hrs}$. & $52.99 *$ & $42.86^{*}$ & $32.04 *$ & $58.62 *$ & $42.70 *$ & $48.18 *$ \\
\hline $10 \mathrm{gm} / 100 \mathrm{ml}$ leaf $48 \mathrm{hrs}$. & $50.00 *$ & $38.96^{*}$ & $29.03 *$ & $56.55^{*}$ & $39.33 *$ & $47.02 *$ \\
\hline $10 \mathrm{gm} / 100 \mathrm{ml}$ leaf $72 \mathrm{hrs}$. & $48.51^{*}$ & $32.47 *$ & $34.67 *$ & $55.86^{*}$ & $35.96^{*}$ & $49.62 *$ \\
\hline $5 \mathrm{gm} / 100 \mathrm{ml}$ bark $24 \mathrm{hrs}$. & $66.42 *$ & $72.73 *$ & $34.07 *$ & $67.59 *$ & $51.69^{*}$ & $61.06^{*}$ \\
\hline $5 \mathrm{gm} / 100 \mathrm{ml}$ bark $48 \mathrm{hrs}$. & $63.43^{*}$ & $70.13 *$ & $28.05^{*}$ & $66.90 *$ & $51.69 *$ & $59.29 *$ \\
\hline $5 \mathrm{gm} / 100 \mathrm{ml}$ bark $72 \mathrm{hrs}$. & $61.94 *$ & $66.23 *$ & $28.16^{*}$ & $65.52 *$ & $50.56^{*}$ & $56.96 *$ \\
\hline $10 \mathrm{gm} / 100 \mathrm{ml}$ bark $24 \mathrm{hrs}$. & 61.94* & $66.23 *$ & $28.16^{*}$ & $63.45 *$ & $48.31 *$ & $54.13 *$ \\
\hline $10 \mathrm{gm} / 100 \mathrm{ml}$ bark $48 \mathrm{hrs}$. & $60.45^{*}$ & $63.64 *$ & $26.80 *$ & $62.76^{*}$ & $46.07 *$ & $54.95^{*}$ \\
\hline $10 \mathrm{gm} / 100 \mathrm{ml}$ bark $72 \mathrm{hrs}$. & $56.72 *$ & $58.44 *$ & 21.94* & $62.76^{*}$ & $43.82 *$ & $57.57 *$ \\
\hline $\begin{array}{c}5 \mathrm{~g} / 100 \mathrm{ml} \text { hot water leaf } \\
\text { extract }\end{array}$ & $55.22 *$ & $41.56^{*}$ & $40.19 *$ & $55.86^{*}$ & $42.70 *$ & $40.77 *$ \\
\hline $\begin{array}{c}10 \mathrm{~g} / 100 \mathrm{ml} \text { hot water leaf } \\
\text { extract }\end{array}$ & $52.24 *$ & $38.96^{*}$ & $35.46^{*}$ & $55.17 *$ & $40.45^{*}$ & $41.86^{*}$ \\
\hline $\begin{array}{c}5 \mathrm{~g} / 100 \mathrm{ml} \text { hot water bark } \\
\text { extract }\end{array}$ & $61.19^{*}$ & $64.94 *$ & $27.46^{*}$ & $57.93 *$ & $47.19 *$ & $40.66^{*}$ \\
\hline $\begin{array}{c}10 \mathrm{~g} / 100 \mathrm{ml} \text { hot water bark } \\
\text { extract }\end{array}$ & $59.70^{*}$ & $62.34 *$ & $26.13 *$ & $57.24 *$ & $44.94 *$ & $41.63 *$ \\
\hline \multicolumn{7}{|c|}{$\begin{array}{l}\text { Each value is the grand mean of five replicates each having ten seeds. Ns= Non-significant, } *=\text { Significant at } 0 \\
<0.05 \\
\text { Table } 4 \text {. Effect of aqueous extracts of } \boldsymbol{C} \text {. lanceolatus on the number of seminal roots of } \\
\text { wheat verities }\end{array}$} \\
\hline \multirow{2}{*}{\multicolumn{3}{|c|}{ Treatments/ extracts }} & \multicolumn{4}{|c|}{ Test species } \\
\hline & & & $\begin{array}{r}\text { T. aestiv } \\
\text { (Cultivar } \mathbf{N}\end{array}$ & $\begin{array}{l}m \\
\text { Illat) }\end{array}$ & $\begin{array}{r}\text { T.aes } \\
\text { (Cultivar }\end{array}$ & $\begin{array}{l}\text { ivum } \\
\text { F. Abad) }\end{array}$ \\
\hline \multicolumn{3}{|c|}{ Control } & \multicolumn{2}{|c|}{$6.63 \pm 1.00$} & $623+100$ & 1.00 \\
\hline \multicolumn{3}{|c|}{$5 \mathrm{gm} / 100 \mathrm{ml}$ leaf $24 \mathrm{hrs}}$. & \multicolumn{2}{|c|}{$4.20 \pm 1.00 *$} & \multicolumn{2}{|c|}{$2.63 \pm 0.97 *$} \\
\hline \multicolumn{3}{|c|}{$5 \mathrm{gm} / 100 \mathrm{ml}$ leaf $48 \mathrm{hrs}}$. & \multicolumn{2}{|c|}{$3.70 \pm 0.99 *$} & \multicolumn{2}{|c|}{$2.20 \pm 0.23 *$} \\
\hline \multicolumn{3}{|c|}{$5 \mathrm{gm} / 100 \mathrm{ml}$ leaf $72 \mathrm{hrs}}$. & \multicolumn{2}{|c|}{$2.23 \pm 0.03^{*}$} & \multicolumn{2}{|c|}{$1.93 \pm 0.29 *$} \\
\hline \multicolumn{3}{|c|}{$10 \mathrm{gm} / 100 \mathrm{ml}$ leaf $24 \mathrm{hrs}}$. & \multicolumn{2}{|c|}{$3.03 \pm 1.00 *$} & \multicolumn{2}{|c|}{$2.53 \pm 0.32 *$} \\
\hline \multicolumn{3}{|c|}{$10 \mathrm{gm} / 100 \mathrm{ml}$ leaf $48 \mathrm{hrs}$. } & \multicolumn{2}{|c|}{$3.03 \pm 0.79 *$} & \multicolumn{2}{|c|}{$2.15 \pm 0.65^{*}$} \\
\hline \multicolumn{3}{|c|}{$10 \mathrm{gm} / 100 \mathrm{ml}$ leaf $72 \mathrm{hrs}}$. & $2.04 \pm 0.0$ & & $1.19 \pm$ & $.10^{*}$ \\
\hline $5 \mathrm{gm} / 100 \mathrm{ml}$ bar & $4 \mathrm{hrs}$. & & $4.80 \pm 0.8$ & & $2.73 \pm$ & $.51^{*}$ \\
\hline $5 \mathrm{gm} / 100 \mathrm{ml}$ bar & $8 \mathrm{hrs}$. & & $3.75 \pm 0.2$ & & $2.63 \pm$ & $.51^{*}$ \\
\hline $5 \mathrm{gm} / 100 \mathrm{ml}$ bar & $2 \mathrm{hrs}$. & & $3.02 \pm 0.2$ & & $2.50 \pm$ & $.51^{*}$ \\
\hline $10 \mathrm{gm} / 100 \mathrm{ml} \mathrm{ba}$ & 24 hrs. & & $3.15 \pm 0.9$ & & $2.20 \pm$ & $1.00^{*}$ \\
\hline $10 \mathrm{gm} / 100 \mathrm{ml} \mathrm{ba}$ & 48 hrs. & & $3.12 \pm 1.0$ & & $2.03 \pm$ & $0.04 *$ \\
\hline $10 \mathrm{gm} / 100 \mathrm{ml} \mathrm{ba}$ & 72 hrs. & & $2.20 \pm 0.6$ & & $1.97 \pm$ & $.01^{*}$ \\
\hline $5 \mathrm{~g} / 100 \mathrm{ml}$ hot water & af extract & & $2.83 \pm 0.0$ & & $2.37 \pm$ & $.79 *$ \\
\hline $10 \mathrm{~g} / 100 \mathrm{ml}$ hot wate & af extract & & $2.50 \pm 0.0$ & & $0.87 \pm$ & $0.03 *$ \\
\hline
\end{tabular}




\begin{tabular}{|c|c|c|}
\hline $5 \mathrm{~g} / 100 \mathrm{ml}$ hot water bark extract & $3.70 \pm 0.80^{*}$ & $2.47 \pm 0.79 *$ \\
\hline $10 \mathrm{~g} / 100 \mathrm{ml}$ hot water bark extract & $3.20 \pm 0.13^{*}$ & $1.47 \pm 0.52 *$ \\
\hline
\end{tabular}

Each value is the grand mean of five replicates each having ten seeds. Ns= Non-significant, ${ }^{*}=$ Significant at $\alpha$ $<0.05$

Table 5. Effect of mulching and litter of C. lanceolatus on the germination and overall growth of wheat verities

\begin{tabular}{|c|c|c|c|c|c|c|c|}
\hline \multirow{2}{*}{$\begin{array}{c}\text { Treatments } \\
\text { Wheat verities }\end{array}$} & \multicolumn{5}{|c|}{ Added Mulch } & \multicolumn{2}{|c|}{ Added litter } \\
\hline & Control & $\begin{array}{l}\text { 5gm } \\
\text { leaf }\end{array}$ & $\begin{array}{c}\text { 10gm } \\
\text { leaf }\end{array}$ & $\begin{array}{c}5 \text { ggm } \\
\text { bark }\end{array}$ & $\begin{array}{l}\text { 10gm } \\
\text { bark }\end{array}$ & Control & 5gm litter \\
\hline \multicolumn{8}{|c|}{ Germination $(\%)$} \\
\hline $\begin{array}{c}\text { T. aestivum } \\
\text { (Cultivar Millat) }\end{array}$ & 100.00 & $90.00 *$ & $52.50 *$ & $90.00^{*}$ & $97.50^{\mathrm{Ns}}$ & 100.00 & $85.00 *$ \\
\hline $\begin{array}{c}\text { T. aestivum } \\
\text { (Cultivar F. Abad) }\end{array}$ & 100.00 & $57.50 *$ & $50.00 *$ & $87.50 *$ & $90.00 *$ & 100.00 & $87.00 *$ \\
\hline \multicolumn{8}{|c|}{ Plumule length (in mm) } \\
\hline $\begin{array}{c}\text { T. aestivum } \\
\text { (Cultivar Millat) }\end{array}$ & 123.00 & $33.60 *$ & $06.45^{*}$ & $85.50 *$ & $90.15^{*}$ & 40.13 & $19.03 *$ \\
\hline $\begin{array}{c}\text { T. aestivum } \\
\text { (Cultivar F. Abad) }\end{array}$ & 81.25 & $17.75^{*}$ & $06.73 *$ & $73.13 *$ & $72.88 *$ & 45.58 & $09.38 *$ \\
\hline \multicolumn{8}{|c|}{ Radicle length (in mm) } \\
\hline $\begin{array}{c}\text { T. aestivum } \\
\text { (Cultivar Millat) }\end{array}$ & 63.63 & $18.73 *$ & $3.85^{*}$ & $45.50 *$ & $40.70 *$ & 40.40 & $20.65^{*}$ \\
\hline $\begin{array}{c}\text { T. aestivum } \\
\text { (Cultivar F. Abad) }\end{array}$ & 51.35 & $11.25^{*}$ & $3.55^{*}$ & $47.50^{*}$ & $36.35^{*}$ & 37.83 & $9.38^{*}$ \\
\hline \multicolumn{8}{|c|}{ Seminal root number } \\
\hline $\begin{array}{c}\text { T. aestivum } \\
\text { (Cultivar Millat) }\end{array}$ & 04.06 & $02.88 *$ & $01.35^{*}$ & $03.83 *$ & $04.23^{\mathrm{ns}}$ & 04.43 & $03.68 *$ \\
\hline $\begin{array}{c}\text { T. aestivum } \\
\text { (Cultivar F. Abad) }\end{array}$ & 03.50 & $02.10 *$ & $00.95^{*}$ & $03.43^{\mathrm{ns}}$ & $02.98 *$ & 04.33 & $02.98 *$ \\
\hline \multicolumn{8}{|c|}{ Fresh weight in $\mathrm{mg}$ (\% of control) } \\
\hline $\begin{array}{r}\text { T. aestivu } \\
\text { (Cultivar Mi }\end{array}$ & & $81.22 *$ & $74.5^{*}$ & $49.01 *$ & $83.21 *$ & \multicolumn{2}{|c|}{$93.43^{*}$} \\
\hline \multicolumn{2}{|c|}{$\begin{array}{c}\text { T. aestivum } \\
\text { (Cultivar F. Abad) }\end{array}$} & $80.33 *$ & $72.63 *$ & $49.41 *$ & $82.25^{*}$ & \multicolumn{2}{|c|}{$97.34 *$} \\
\hline \multicolumn{8}{|c|}{ Dry weight in $\mathrm{mg}$ (\% of control) } \\
\hline $\begin{array}{r}\text { T. aestivu } \\
\text { (Cultivar Mi }\end{array}$ & & $66.04 *$ & $75.08^{*}$ & $32.09^{*}$ & $68.54 *$ & \multicolumn{2}{|c|}{$49.75^{*}$} \\
\hline \multicolumn{2}{|c|}{$\begin{array}{c}\text { T. aestivum } \\
\text { (Cultivar F. Abad) }\end{array}$} & $67.47 *$ & $75.60 *$ & $32.83 *$ & $68.98^{*}$ & \multicolumn{2}{|c|}{$51.24 *$} \\
\hline \multicolumn{8}{|c|}{ Moisture content (\% of control) } \\
\hline \multicolumn{2}{|c|}{$\begin{array}{c}\text { T. aestivum } \\
\text { (Cultivar Millat) }\end{array}$} & $81.21 *$ & $72.38 *$ & $47.68 *$ & $83.27 *$ & \multicolumn{2}{|c|}{$97.07 *$} \\
\hline \multicolumn{2}{|c|}{$\begin{array}{c}\text { T. aestivum } \\
\text { (Cultivar F. Abad) }\end{array}$} & $80.04 *$ & $70.17 *$ & $48.07 *$ & $82.12 *$ & \multicolumn{2}{|c|}{$98.44^{*}$} \\
\hline
\end{tabular}

Each value is the grand mean of five replicates each having ten seeds. Ns= Non-significant, $*=$ Significant at $\alpha$ $<0.05$

\section{Conclusion}

The present study suggested that Callistemon lanceolatus DC. has robust allelopathic potential in contradiction of the test crops. Allelopathic stress significantly affected the germination and overall growth of the test crops. In fields, it is relatively probable that the $C$. lanceolatus might be one of the thinkable reasons of decrease of wheat yields due to 
its allelopathy. Therefore, its plantation should be prudently assessed along the crop field. Furthermore, field experiments are required to confirm these results.

\section{Authors' contributions}

Conceived and designed the experiments: N Shah, I Ahmad \& M Imran, Performed the experiments: N Shah \& M Imran, Analyzed the data: H Gul \& MJ Babar, Contributed materials/ analysis/ tools: S Anwar \& H Gul, Wrote the paper: N Shah, M Imran, WA Shah \& S Anwar.

\section{References}

1. Caton BP, Mortimer AM, Hill TC, Gibson JE \& Fisher AJ (1999). Weed morphology effects on competitiveness for light in direct-seeded rice. Proc 17th Asian-Pacific, weed Science Society Conference Bangkok 1: 116-120.

2. Hussain F, Ahmad B \& Ilahi I (2010). Allelopathic effects of Cenchrus ciliaris L., and Bothriochloa pertusa (L.) A. Camus. Pak J Bot 42(5): 3587-3604. Hussain F, Ilahi I, Malik SA, Dasti AA \& Ahmad B (2011). Allelopathic effects of rain leachates and roots exudates of Cenchrus ciliaris L., and Bothriochloa pertusa (L.) A. Camus. Pak J Bot 43(1): 341-350.

3. Tabaglio V, Gavazzi C, Schulz M \& Marocco A (2008). Alternative weed control using the allelopathic effect of natural benzoxazinoids from rye mulch. Agron Sustain Dev 28(3): 397-401.

4. Uremis I, Arslan M, Uludag A \& Sangun MK (2009). Allelopathic potentials of residues of 6 Brassica species on Johnson grass Sorghum halepense (L.) Pers. African J Biotech 8(15): 3497-3501.

5. Hussain F \& Abidi N (1991). Allelopathy exhibited by Imperata cylindrical (L.) P. beauv. Pak J Bot 23(1): 15-25.

6. Rebaz Z, Shaukat SS \& Siddiqui IA (2001). Allelopathic potential of anagllis arvensis L. A cosmopolitan weed. Pak J Biol Sci 4(4): 446-450.

7. Sahar A, Khawas E \& Shehata M (2005). Allelopathic potentialities of acacia nilotica and eucalyptus rostrata on monocot (Zea mays L.) and dicot
(Phaseolus vulgaris L.) Biotechnology 4(1): 23-34.

8. Maharjan S, Shrestha BB \& Jha PK (2007). Allelopathic effects of aqueous extract of leaves of Parthenium hysterophorus on seed germination and seedling growth of some cultivated and wild herbaceous species. Scientific World 5(5): 33-39.

9. Siddiqui S, Yadav R, Yadav K, Wani FA, Meghvansi MK, Sharma S \& Jabeen F (2009). Allelopathic potentialities of different concentration of aqueous leaf extracts of some Arable trees on germination and radicle growth of Cicer arietinum Var. C-235. Global J Molecular Science 4(2): 91-95.

10. Abugre S, \& Sam QSJ (2010). Evaluating the Allelopathic Effect of Jatropha curcas aqueous extract on Germination, Radicle and Plumule length of Crops. Inter J Agri Biol 12: 769-772.

11. Kavitha D, Prabhakaran J \& Arumugam K (2012). Allelopathic influence of Vitex negundo L., on germination and growth of Green gram Vigna radiate (L.) R. Wilczek. and Black gram Vigna mungo (L.) Hepper. Int J Ayur Herb Med 2(1): 163-170.

12. Nekonam MS, Razmjoo J, Sharifnabi B \& Karimmojeni H (2013). Assessment of allelopathic plants for their herbicidal potential against field bindweed (Convolvulus arvensis). A J C S 7(11): 1654-1660.

13. Ahmad I, Hussain F, Barkatullah \& Ahmad B (2014). Phytotoxic potential of celtis australis L. against four crop species. Pak J Bot 46(6): 2063-2067.

14. Kalpana $P$ \& Navin MK (2015). Assessment of Allelopathic Potential of Leucaena leucocephala (Lam) De Vit on Raphanus sativus L. Inter J Scienti Res Pub 5(1): 1-3.

15. Hussain S, Siddiqu S, Khalid S, Jamal A, Qayyum A \& Ahmad Z (2007). Allelopathic potential of Senna Cassia angustifolia Vahl., on germination and seedling characters of some major cereal crops and their associated grassy weeds. Pak J Bot 39(4): 1145-1153. 
16. Elizabeth GB, Pivello VR \& Meirelles ST (2008). Allelopathic evidence in Brachiaria decumbens and its potential to invade the Brazilian Cerrados. Braz Arch Biol Techn 51(4): 825-831.

17. Hussain F \& Ilahi I (2009). Allelopathic potential of Cenchrus ciliaris L., and Bothriochloa pertusa (L.) A. Camus. $J$ Sci Techn 33(2): 47-55.

18. Patel B, Achariya B \& Bupripata NP (2002). Allelopathic effects of eucalyptus leaves on seed germination and seedling growth of winter wheat. Proc Ind Soc Allelopathy Pp. 115-119.

19. Nadal DPS, Birta SS \& Narwal SS (2005). Allelopathic influence of eucalyptus litter on germination, yield and yield components of live wheat varieties. Proc 1st Nat Symp on Allelopathy in Agric Systems.

20. Khan MA, Hussain I \& Khan EA (2007). Effect of aqueous extract of Eucalyptus camaldulensis L., on germination and growth of maize (Zea mays L.). Pak J Weed Sci Res 13(3-4): 177-182.

21. Kumar M \& Siangshai S (2009). Effect of leaf and bark aqueous extracts on germination and radicle length of crops in Mizoram. Pak J Weed Sci Res 15(4): 275-281.

22. Todaria NP, Singh B \& Dhanai CS (2005). Allelopathic effects of tree extract, on germination and seedling growth of field crops. Allelopathy $J$ 15(2): 285-294.

23. Hussain F, Niaz F, Jabeen M \& Burni T (2004). Allelopathic potential of Broussonetia papyrifera Vent. Pak J Pl Sci 10(2): 69-78.

24. Sher Z, Hussain F, Ahmad B \& Wahab M (2011). Allelopathic potential of Populus euphratica Oliver. Pak J Bot 43(4): 1899-1903.

25. Khan MA, Hussain I \& Khan EA (2008). Allelopathic effects of Eucalyptus (Eucalyptus camaldulensis L.) on germination and seedling growth of wheat (Triticum aestivum L.). Pak $J$ Weed Sci Res 14(1-2): 9-18.

26. Barkatullah, Hussain $F$ \& Ibrar $M$ (2010). Allelopathic potential of Dodonaea viscosa (L.) Jacq Pak J Bot 42(4): 2383-2390.

27. Pervez SS, Pervez MM, Nishihara E, German H \& Fujii Y (2003). Tamarindus indica L., leaf is a source of allelopathic substance. Plant Growth Regulator 40(2):107-115.

28. Samreen U, Hussain F \& Sher Z (2009). Allelopathic potential of Calotropis procera (Ait.) Ait. Pak J Pl Sci 15(1): 7-14.

29. Yang CM, Chang IF, Lin SJ \& Chou CH (2002). Effects of three allelopathic phenolics on chlorophyll accumulation of rice (Oryza sativa L.) seedlings inhibition of supply orientation. Bot Bull Acad Sinica 43: 299-304.

30. Alagesaboopthi C (2010). Allelopathic effects of Centella asiatica aqueous extracts on pearl millet (Pennisetum typhoides L.) and cowpea (Vigna unguiculata Walp.) Pak J Weed Sci Res 16(1): 67-71.

31. Uniyal AK \& Chhetri S (2010). An assessment of phytotoxic potential of promising agroforestry treason germination and growth pattern of traditional field crops of Sikkim Himalaya, India. American-Eurasian $J$ Agri Envi Sci 9: 70-78.

32. Inderjit $S$ \& Duke O (2003). Eco physiological aspects of allelopathy. Planta 217(4): 529-539.

33. Sarah S, Hussain F, Ehsan M \& Burni $T$ (2011). Allelopathic potential of Polypogon monspeliensis L., against two cultivars of wheat. African $J$ Biotech 10(85): 19723-1972.

34. Sarah S, Hussain F, Ehsan M \& Burni $\mathrm{T}$ (2011). Allelopathic potential of Polypogon monspeliensis L., against two cultivars of wheat. Afr J Biotch 10(85): 19723-1972. 